\title{
On the Intermediate Line Region in AGNs
}

\author{
T. P. Adhikari ${ }^{1, *}$, A. Różańska ${ }^{1}$, K. Hryniewicz ${ }^{1}$, B. Czerny ${ }^{1,2}$, G.J. Ferland ${ }^{3}$ \\ ${ }^{1}$ Nicolaus Copernicus Astronomical Center, Polish Academy of Sciences, \\ Bartycka 18, 00-716, Warsaw, Poland \\ ${ }^{2}$ Center for Theoretical Physics, Polish Academy of Sciences, Aleja Lotnikow \\ 32/46, Warsaw, Poland \\ ${ }^{3}$ Department of Physics and Astronomy, The University of Kentucky, Lexington, $K Y$ \\ 40506, USA
}

Correspondence*:

T. P. Adhikari

tek@camk.edu.pl

\begin{abstract}
In this paper we explore the intermediate line region (ILR) by using the photoionisation simulations of the gas clouds present at different radial distances from the center, corresponding to the locations from BLR out to NLR in four types of AGNs. We let for the presence of dust whenever conditions allow for dust existence. All spectral shapes are taken from the recent multi-wavelength campaigns. The cloud density decreases with distance as a power law. We found that the slope of the power law density profile does not affect the line emissivity radial profiles of major emission lines: $\mathrm{H} \beta, \mathrm{He} \mathrm{II}, \mathrm{Mg} \mathrm{II}, \mathrm{C} \mathrm{III]} \mathrm{and} \mathrm{[O} \mathrm{III].} \mathrm{When} \mathrm{the} \mathrm{density} \mathrm{of} \mathrm{the}$ cloud at the sublimation radius is as high as $10^{11.5} \mathrm{~cm}^{-3}$, the ILR should clearly be seen in the observations independently of the shape of the illuminating radiation. Moreover, our result is valid for low ionization nuclear emission regions of active galaxies.
\end{abstract}

Keywords: active galaxies, emission lines, photoionisation, radiative transfer, accrection disk

\section{INTRODUCTION}

The emission lines in active galactic nuclei (AGNs) provide a unique opportunity to study the properties of the materials located in the environment of the supermassive black hole (SMBH). From the measurement of full width at half maxima (FWHM) of lines in the observed AGN spectra, it is well understood that there exist two separate regions of the line emission. Lines with FWHM $\geq 3000 \mathrm{~km} \mathrm{~s}^{-1}$ are emitted by materials with densities $\sim 10^{10} \mathrm{~cm}^{-3}$ in the broad line region (BLR) located closer to the AGN central engine. Whereas, the narrow line region (NLR) with gas densities $\sim 10^{5} \mathrm{~cm}^{-3}$, located much farther from the AGN center, emits the narrow lines with FWHM $\sim 500 \mathrm{~km} \mathrm{~s}^{-1}$. A clear spatial separation in between BLR and NLR is present where the emission of lines with intermediate FWHM is not seen in the observations.

Theoretically, the lack of emission from the intermediate line region (ILR) was explained by Netzer and Laor (1993, hereafter NL93) as an effect of dust extinction, both in absorption and scattering of line photons and continuum. The authors considered radially distributed clouds of different density and ionization level. Broad and narrow line regions were separated due to the dust content which cannot 
be present in BLR since the gas temperatures are so high that the dust grains cannot survive there. Nevertheless, further out from the nucleus there is a boundary radius named sublimation radius, $R_{\mathrm{d}}$, where temperature drops substantially, and dust can sustain up to the distances where NLR is located. NL93 presented that, for assumed gas parameters, the strong drop of line emission appears at distances where potential ILR is expected. Therefore natural separation between BLR and NLR occurs when the dust is taken into account in photoionisation calculations of cloud's emission. This natural separation disclaims the existence of the ILR. In the ILR, the ionisation parameter is higher than in further located NLR clouds and hence the relative effect of the dust absorption is stronger. The dust suppressed emission in ILR reappears on transition to the NLR when dust absortion becomes negligible due to the low gas temperature. However, in the recent observations of some AGNs, additional intermediate line component of FWHM $\sim 700-1200 \mathrm{~km} \mathrm{~s}^{-1}$ is clearly required to fit the lines in their emission spectra (Brotherton et al., 1994; Mason et al., 1996; Crenshaw and Kraemer, 2007; Hu et al., 2008a, b; Zhu et al., 2009; Li et al., 2015). The open questions are: does the ILR exist physically separated from BLR and NLR? What are the mechanisms that give rise to ILR in some sources but not in others?

Recently, Adhikari et al. (2016, hereafter AD16) have shown, using the framework put forward by NL93, that when the density of illuminated clouds is high enough, the dust does not suppress the gap between BLR and NLR and intermediate line emission is clearly visible. The authors performed photoionisation simulations of radially distributed clouds subject to the radiation of four different spectral energy distributions (SEDs), most common types of AGN. The dust content was introduced at the sublimation radius of assumed value: $R_{\mathrm{d}}=0.1 \mathrm{pc}$. In NL93, the authors assumed a power law with slope -1.5 and normalization $10^{9.4} \mathrm{~cm}^{-3}$ at $R_{\mathrm{d}}$ to describe the variation of the density of gas clouds with distance from the nucleus. These clouds were then illuminated by the mean AGN spectrum. Resulting emissivity profiles contained the suppression of line emission between BLR and NLR as it is commonly observed. AD16 made one step forward, showing that if the density at the sublimation radius is high, of the order of $10^{11.5} \mathrm{~cm}^{-3}$, the ILR is clearly visible. Such result appeared to be independent on SED of illuminated radiation taken into account. Additionally, the authors argued that the low ionization nuclear emission regions (LINERs) should also exhibit the ILR.

In this paper, we expand the work of AD16 and investigate the variation of density profile of radially distributed clouds. All photoionisation simulations are done with the most recent version of the CLOUDY code (Ferland et al., 2017). To accommodate broad types of ionising SEDs in our calculations, we considered four distinct AGN types: Sy1.5 galaxy Mrk 509 (Kaastra et al., 2011), Sy1 galaxy NGC 5548 (Mehdipour et al., 2015), NLSy1 galaxy PMN J0948+0022 (D’Ammando et al., 2015), and LINER NGC 1097 (Nemmen et al., 2014), each of them obtained from currently available simultaneous multi-wavelength observations. As a result of our simulations, we derived the line emissivity radial profiles for major emission lines: $\mathrm{H} \beta \lambda 4861.36 \AA$, He II $\lambda 1640.00 \AA, \mathrm{Mg}$ II $\lambda 2798.0 \AA$, C III] $\lambda 1909.00 \AA$ and [O III] $\lambda 5006.84 \AA$.

Adopting the density normalization to be $10^{11.5} \mathrm{~cm}^{-3}$ at $R_{\mathrm{d}}=0.1 \mathrm{pc}$, all the power law density distribution yield continuous line emissivity profiles with prominent intermediate line emission component in permitted lines $\mathrm{H} \beta$, He II and $\mathrm{Mg}$ II, independent of the density slopes and the spectral radiation shapes adopted. Below we briefly outline the photoionisation model itself, and discuss the resulting line emissivity profiles focusing mainly on the visibility of ILR in different AGN. 


\section{PHOTOIONISATION MODEL}

The simulation of the photoionisation process is done with the publicly available numerical code CLOUDY version c17 (Ferland et al., 2017), which takes into account all the relevant radiative processes when a gas cloud is subjected to an incident radiation field. A simplistic geometrical set up of gas distributed form BLR further out to NLR is arranged by assuming spherical clouds with varying gas density, $n_{\mathrm{H}}$, and the total column density $N_{\mathrm{H}}$, at each radial distances, $r$, from the SMBH:

$$
n_{\mathrm{H}}(r)=10^{11.5}\left(r / R_{\mathrm{d}}\right)^{-\beta}, \quad N_{\mathrm{H}}(r)=10^{23.4}\left(r / R_{\mathrm{d}}\right)^{-1}
$$

where $\beta$ is the power law density slope, and $R_{\mathrm{d}}$ is arbitrarily chosen to be equal $0.1 \mathrm{pc}$ (following NL93 and AD16). The total column density of a cloud located at the sublimation radius is assumed after NL93: $N_{\mathrm{H}}\left(\right.$ at $\left.\mathrm{R}_{\mathrm{d}}\right)=10^{23.4} \mathrm{~cm}^{-2}$, and the gas hydrogen density after AD16 $: n_{\mathrm{H}}\left(\right.$ at $\left.\mathrm{R}_{\mathrm{d}}\right)=10^{11.5} \mathrm{~cm}^{-3}$. Here, we stress that the density normalizations lower than the value adopted in this paper, do not reproduce the intermediate line emission as shown by AD16.

Besides three previously considered types of AGN incident radiation shapes: Sy1.5 galaxy Mrk 509 (Kaastra et al., 2011), Sy1 galaxy NGC 5548 (Mehdipour et al., 2015), NLSy1 PMN J0948+0022 (D'Ammando et al., 2015), in this paper we also used the shape appropriate for LINER NGC 1097 (Nemmen et al., 2014). This choice of SED covers the general shapes of the radiation emanating from the AGN central engine. Adopted SEDs are the incident spectra used in the photoionisation simulation, where clouds distributed along the range of radii are exposed to the same type of radiation. All the SEDs are normalized to the bolometric luminosity $10^{45} \mathrm{erg} \mathrm{s}^{-1}$ which is an input to the CLOUDY code. This allows to compute the ionizing flux i.e. ionization parameter at each cloud radius.

We adopted the CLOUDY default chemical abundances, which are mostly the Solar values derived by Grevesse and Sauval (1998) for the gas clouds at $r \leq R_{\mathrm{d}}$, whereas the interstellar medium (ISM) composition 1 with dust grains is used for the clouds at $r>R_{\mathrm{d}}$. This assumption is consistent with the observational suggestions that the BLR is devoid of dust whereas the lower temperature in NLR allows its existence. On moving further out from BLR to NLR, the increase in radial distance is accompanied by the decrease in ionization degree and a cloud thickness.

The aim of this paper is to search how the appearance of intermediate line emission is sensitive on the value of density power law slope. Below we present line luminosity radial profiles for six values of $\beta$ $=0.5,1.0,1.5,2.0,2.5,3.0$. We are aware that the power law density distribution of clouds does not reflect realistic situation in AGN, but it is sufficient for the purpose of this paper. In the forthcoming paper (Adhikari et al., 2017, in preparation), we plan to use realistic density profile, which is expected where clouds form from outflowing gas above the accretion disk atmosphere. Furthermore, we plan to consider measured values of bolometric luminosities, which give the realistic position of sublimation radius for each type of AGN due to the formula by Nenkova et al. (2008).

\section{LINE EMISSIVITIES}

As the results of photoionisation simulations, we compute line luminosities emitted from clouds located at the each radii. Therefore, by presenting line luminosity dependence on the distance from SMBH, i.e. line luminosity radial profile, we can check if the emission from ILR is comparable to the BLR and NLR or substantially lower. This is our basic test for the existence of ILR in all considered types of AGN.

\footnotetext{
1 for details see the Hazy1 CLOUDY documentation.
} 
We derived the line luminosity radial profiles for the major emission lines : $\mathrm{H} \beta \lambda 4861.36 \AA$, He II $\lambda 1640.00 \AA, \mathrm{Mg}$ II $\lambda 2798.0 \AA, \mathrm{C} \mathrm{III]} \lambda 1909.00 \AA$ and [O III] $\lambda 5006.84 \AA$. The resulting line emissivity profiles for the four cases of SED are presented in the Fig. 1 and Fig. 2, In all cases of density power law slopes, we recovered a continuous line emission, with a small enhancement of the permitted lines $\mathrm{H} \beta$ and $\mathrm{He}$ II at the radial distance around $0.1 \mathrm{pc}$ corresponding to the intermediate region, independent of the shape of the SEDs in consideration. There is a small reduction of $\mathrm{Mg}$ II line at $0.1 \mathrm{pc}$ though not very significant as compared to the suppression presented by NL93. The semi forbidden line C III] contribution to the intermediate emission component becomes the most prominent for the density profile with $\beta=1.5$. These results corroborate with the conclusion of AD16 that when the density of the emitting gas is high enough, the extinction effect of dust grains on line production is negligible.
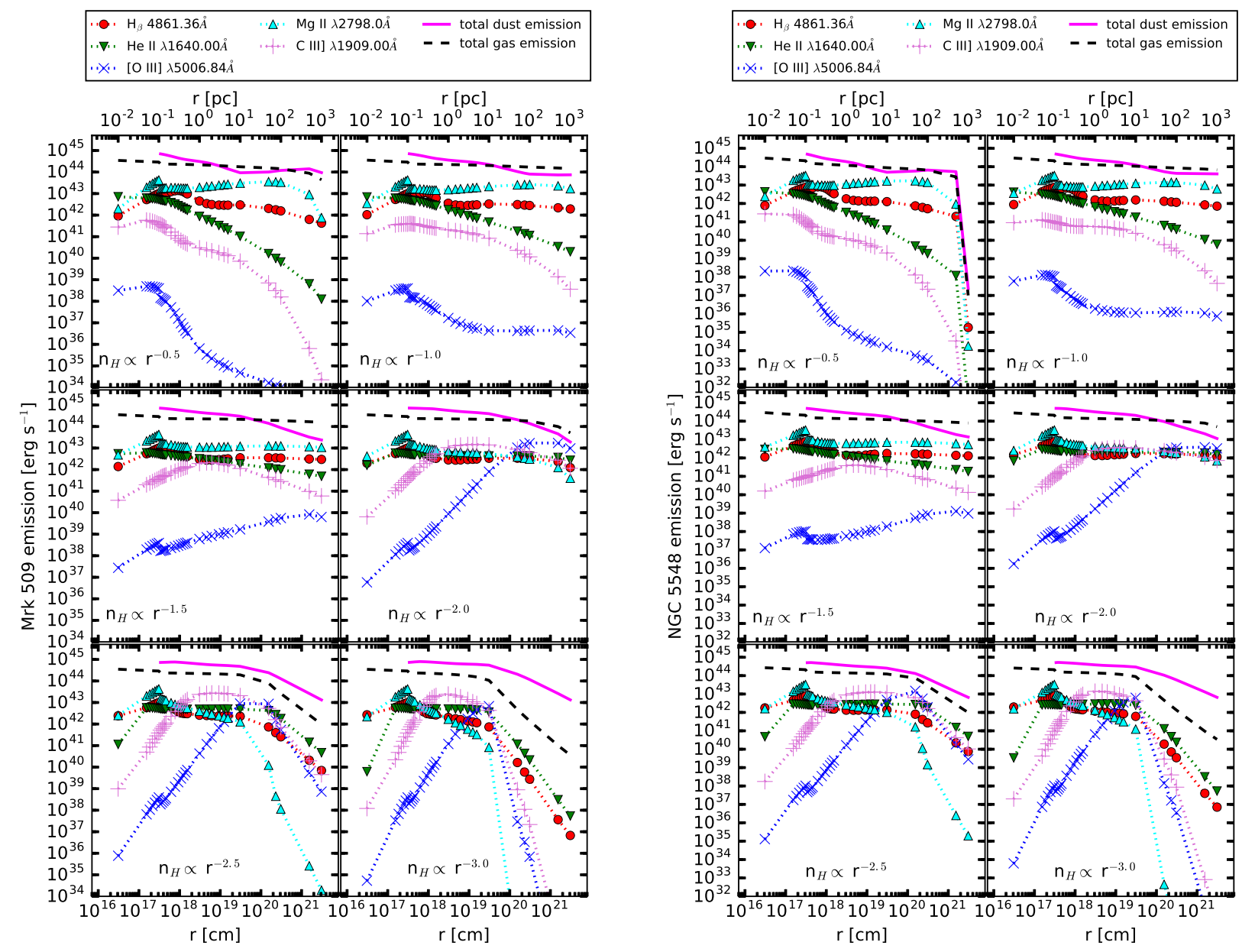

Figure 1. Left panel: Line emission versus radius for Mrk 509 SED. Different subplots are for various density slopes given in the left corners. Major emission lines: $\mathrm{H} \beta$ (red circles), He II (green triangles down), [O III] (blue crosses), Mg II (cyan triangles up) and C III] (magenta pluses) are shown for each density profile cases. For clarity total dust emission (magenta continuous line) and total gas emission (black dashed line) are also shown. Right panel: The same as in left panel but with the spectral radiation shape of NGC 5548

The most noticeable effects of different density profiles on line emissivities occur in the NLR range, i.e. for $r>50 \mathrm{pc}$. This behavior is quite obvious since for those radii, differences in densities between profiles are the biggest. For $\beta=0.5$ and 1.0, density falls slowly and remains moderately high across the radii causing the strong suppression of forbidden line [O III]. [O III] is effectively produced in low density 
environment and becomes prominent when the density around the radius $10 \mathrm{pc}$ becomes low enough, the cases for the profiles with $\beta \geq 2.0$. Narrow line emission is dominated by the C III] components when the density distribution is given by the profiles with $\beta \geq 2.0$. We found that the derived line emissivities for all cases of power law density slopes, particularly in the region of intermediate line emission, do not strongly depend on the shapes of the SED used. There are subtle differences in emissivities corresponding to the BLR and NLR due to the different amount of UV and soft X-ray photons among the SEDs. This result is in agreement with the conclusion of AD16, that the presence of ILR emission is not determined by the shape of the incident radiation.
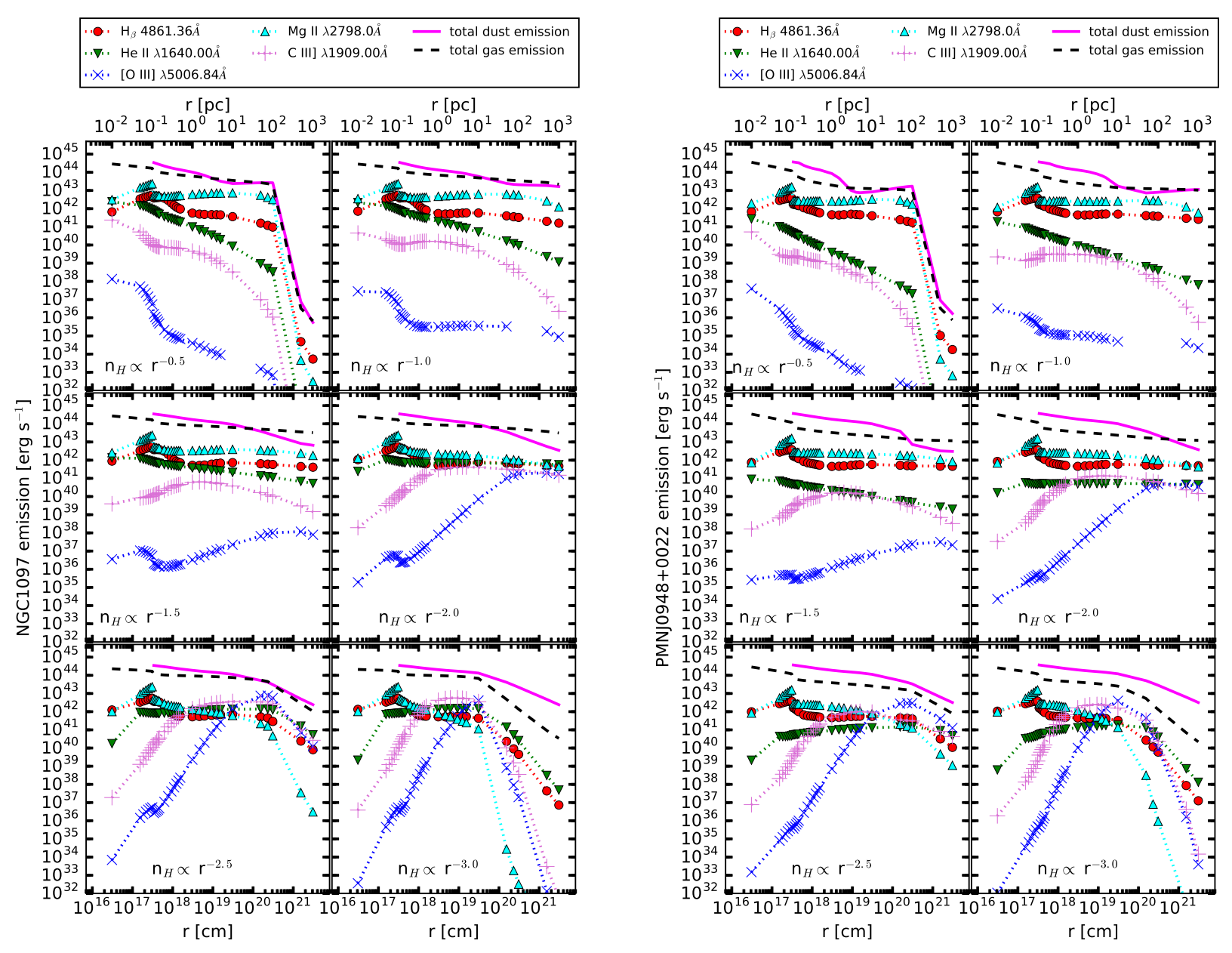

Figure 2. The same as in Fig. 1 but for the SED of NGC 1097 (left panel) and PMN J0948+0022 (right panel).

\section{DISCUSSION}

The results above confirm the conclusion of AD16, that the dust extinction of the emission lines in AGN introduced by NL93 is important only when the gas density is low. In AD16, the authors adopted only a density power law of slope $\beta=1.5$ whereas this work demonstrates that the different slopes of the density distribution do not matter significantly as long as the gas density at the sublimation radius is high, in this case being $10^{11.5} \mathrm{~cm}^{-3}$. In all cases of considered density profiles, we obtained an intermediate line emission around 0.1-1 pc, mostly manifested in permitted lines $\mathrm{H} \beta$, He II and $\mathrm{Mg}$ II, and weakly present in the semi forbidden line C III]. This indicates that the high density and the low ionisation environment 
favors the intermediate line emission rather than the high ionization environment where the forbidden line [O III] is produced. So, in the AGNs where the ILR is seen in observations, the emitting region is composed of the dense and less ionized gas.

The physical reason for the disappearance of the effect of dust is connected with the size of $\mathrm{H}$ - ionized front in the gas. At very high value of ionization parameter, i.e. for the low density case, the volume of the H-ionized region is very large, if not the full cloud volume. When the density of cloud increases, the ionization decreases, and a cloud consists of two regions: $\mathrm{H}$-ionized region and $\mathrm{H}$-neutral region. The line emission comes from the $\mathrm{H}$-ionized region, and only the dust in this region competes with the gas for the photons. In other words, the dense clouds have much smaller geometrical thickness of $\mathrm{H}$-ionized layer, smaller dust column density in the region with abundant photons, and therefore the dust absorption is negligible. Our simulations are not yet aimed to make a quantitative statements about the studied objects. For that, we would need to do more extensive study, representing the bolometric luminosity and the position of the inner radius of the dust distribution appropriate for a given object. However, the grid of results shows a clear trend.

In the recent years, there has been promising claims that, broad line emission clouds in AGN are connected with the wind from the upper part of an accretion disk atmosphere (Gaskell, 2009; Czerny and Hryniewicz, 2011). As shown in the Fig. 6 of AD16, the density profiles computed in the upper part of the standard disk atmosphere can be quite dense with values up to $\sim 10^{15} \mathrm{~cm}^{-3}$ at the assumed position of sublimation radius. Those density values depend on the mass of the black hole and the disk accretion rate. Because of the high gas density, photoionisation simulations outcome with continuous line luminosity radial profile for the reason discussed in the previous paragraph. As the consequence, ILR should be observed together with BLR and NLR. The use of realistic density profiles expected from the accretion disk atmosphere is very important in the aim to understand the nature and origin of the ILR observed in some AGNs. This work is in progress and will be presented by Adhikari et al. (2017, in preparation).

\section{CONFLICT OF INTEREST STATEMENT}

The authors declare that the research was conducted in the absence of any commercial or financial relationships that could be construed as a potential conflict of interest.

\section{AUTHOR CONTRIBUTIONS}

TPA, AR and KH were responsible for developing the idea, doing the simulations, analysing the results and writing the text for the manuscript, BC provided the concept and GJF discussed the results.

\section{FUNDING}

This research was supported by Polish National Science Center grants No. 2016/21/N/ST9/03311, 2015/17/B/ST9/03422, 2015/18/M/ST9/00541, 2015/17/B/ST9/03436, and by Ministry of Science and Higher Education grant W30/7.PR/2013. It received funding from the European Union Seventh Framework Program (FP7/2007-2013) under the grant agreement No.312789. TPA received funding from NCAC PAS grant for young researchers. 


\section{REFERENCES}

Adhikari, T. P., Hryniewicz, K., Różańska, A., and Czerny (2017). Intermediate line emission in AGN: the effect of gas density prescription. In preperation

Adhikari, T. P., Różańska, A., Czerny, B., Hryniewicz, K., and Ferland, G. J. (2016). The Intermediateline Region in Active Galactic Nuclei. ApJ 831, 68. doi:10.3847/0004-637X/831/1/68

Brotherton, M. S., Wills, B. J., Francis, P. J., and Steidel, C. C. (1994). The intermediate line region of QSOs. ApJ 430, 495-504. doi:10.1086/174425

Crenshaw, D. M. and Kraemer, S. B. (2007). Mass Outflow from the Nucleus of the Seyfert 1 Galaxy NGC 4151. ApJ 659, 250-256. doi:10.1086/511970

Czerny, B. and Hryniewicz, K. (2011). The origin of the broad line region in active galactic nuclei. $A \&$ A 525, L8. doi:10.1051/0004-6361/201016025

D’Ammando, F., Orienti, M., Finke, J., Raiteri, C. M., Hovatta, T., Larsson, J., et al. (2015). The most powerful flaring activity from the NLSy1 PMN J0948+0022. MNRAS 446, 2456-2467. doi:10.1093/ mnras/stu2251

Ferland, G. J., Chatzikos, M., Guzmán, F., Lykins, M. L., van Hoof, P. A. M., Williams, R. J. R., et al. (2017). The 2017 Release of Cloudy. ArXiv e-prints

Gaskell, C. M. (2009). What broad emission lines tell us about how active galactic nuclei work. NAR 53, 140-148. doi:10.1016/j.newar.2009.09.006

Grevesse, N. and Sauval, A. J. (1998). Standard Solar Composition. Space Sci. Rev 85, 161-174. doi:10. 1023/A:1005161325181

Hu, C., Wang, J.-M., Ho, L. C., Chen, Y.-M., Bian, W.-H., and Xue, S.-J. (2008a). H $\beta$ Profiles in Quasars: Evidence for an Intermediate-Line Region. ApJL 683, L115. doi:10.1086/591848

Hu, C., Wang, J.-M., Ho, L. C., Chen, Y.-M., Zhang, H.-T., Bian, W.-H., et al. (2008b). A Systematic Analysis of Fe II Emission in Quasars: Evidence for Inflow to the Central Black Hole. ApJ 687, 78-96. doi:10.1086/591838

Kaastra, J. S., Petrucci, P.-O., Cappi, M., Arav, N., Behar, E., Bianchi, S., et al. (2011). Multiwavelength campaign on Mrk 509. I. Variability and spectral energy distribution. A\&A 534, A36. doi:10.1051/ 0004-6361/201116869

Li, Z., Zhou, H., Hao, L., Wang, H., Ji, T., Shi, X., et al. (2015). Detection of the Intermediate-width Emission Line Region in Quasar OI 287 with the Broad Emission Line Region Obscured by the Dusty Torus. ApJ 812, 99. doi:10.1088/0004-637X/812/2/99

Mason, K. O., Puchnarewicz, E. M., and Jones, L. R. (1996). The origin of the optical emission lines in the narrow-line Seyfert 1 galaxy RE J1034+396. MNRAS 283, L26-L29. doi:10.1093/mnras/283.1.L26

Mehdipour, M., Kaastra, J. S., Kriss, G. A., Cappi, M., Petrucci, P.-O., Steenbrugge, K. C., et al. (2015). Anatomy of the AGN in NGC 5548. I. A global model for the broadband spectral energy distribution. A\&A 575, A22. doi:10.1051/0004-6361/201425373

Nemmen, R. S., Storchi-Bergmann, T., and Eracleous, M. (2014). Spectral models for low-luminosity active galactic nuclei in LINERs: the role of advection-dominated accretion and jets. MNRAS 438, 2804-2827. doi:10.1093/mnras/stt2388

Nenkova, M., Sirocky, M. M., Ivezić, Ž., and Elitzur, M. (2008). AGN Dusty Tori. I. Handling of Clumpy Media. ApJ 685, 147-159. doi:10.1086/590482

Netzer, H. and Laor, A. (1993). Dust in the narrow-line region of active galactic nuclei. ApJL 404, L51-L54. doi:10.1086/186741 
Zhu, L., Zhang, S. N., and Tang, S. (2009). Evidence for an Intermediate Line Region in Active Galactic Nuclei's Inner Torus Region and its Evolution from Narrow to Broad Line Seyfert I Galaxies. ApJ 700, 1173-1189. doi:10.1088/0004-637X/700/2/1173 